\title{
Portales web para gestionar información y potenciar la cocreación de experiencias ${ }^{*}$
}

\author{
\begin{tabular}{|} 
Liliana González Palacio ${ }^{* * *}$ \\
Mauricio González Palacio \\
Manuel Enrique Anaya Taboada ${ }^{* * * * *}$ \\
Mario Alberto Luna-del Risco \\
Isabel Díaz-Forero
\end{tabular}
}

Recibido: 02/11/2017 - Aceptado: 04/07/2018

https://doi.org/10.22395/rium.v18n34a8

\begin{abstract}
Resumen
Múltiples estrategias para potenciar la innovación abierta se han conjugado buscando la generación conjunta de valor entre diversos actores a través de la cocreación de experiencias. Algunas consisten en técnicas presenciales en las que los interesados deben estar concentrados en el mismo lugar y tiempo, mientras otras usan las redes sociales y portales web porque facilitan la colaboración y cocreación de una manera natural y además proporcionan herramientas especializadas de acuerdo a los requerimientos particulares de un proyecto. El presente trabajo muestra un marco de referencia para la selección de un portal web con fines de cocreación, facilitando así la labor de escoger, con criterios claros, la mejor herramienta para un proceso de innovación abierta virtual. Algunos resultados preliminares permiten evidenciar que la selección adecuada de la herramienta de interacción en un proceso de innovación abierta es determinante para el éxito del proceso. De otro lado, se ratifica que el internet (puntualmente herramientas como los portales empresariales de innovación) es un potenciador en el intercambio de información. Lo anterior porque facilita la interacción entre participantes distribuidos geográficamente y ofrece posibilidades de colaborar de forma asíncrona. Con esto se eliminan las limitantes de tiempo y espacio que se presentan al pensar en esquemas presenciales para cocrear.

Palabras clave: tecnologías de información y comunicación; innovación abierta; portales web; cocreación.
\end{abstract}

Este artículo se deriva de un proyecto de investigación cofinanciado por la Universidad de Medellín, Colciencias y la empresa Kuepa, en el marco de la convocatoria 804 de 2018.

** Ph.D. en Ingeniería. Profesora asociada. Ingeniería de Sistemas. Universidad de Medellín, Medellín, Colombia. Carrera 87 n. ${ }^{\circ}$ 30-65. Teléfono: 3405555, extensión 5529. Orcid: http://orcid.org/0000-0002-6029-400X. Correo electrónico: ligonzalez@udem.edu.co

*** M.Sc. en Ingeniería. Profesor auxiliar. Ingeniería de Telecomunicaciones. Universidad de Medellín, Medellín, Colombia. Carrera 87 n. 30-65. Teléfono: 3405555, extensión 5639. Correo electrónico: magonzalez@udem. edu.co. Orcid: http://orcid.org/0000-0002-6727-7472

***** Ingeniero de Sistemas. Helmes, Tallin, Estonia. Lõõtsa 6B 11415. Teléfono: +372 6106100. Correo electrónico: manuel.anaya@helmes.com. Orcid ID: http://orcid.org/0000-0002-0384-5338

***** Ph.D. en conservación ambiental. Profesor asistente. Ingeniería en Energía. Universidad de Medellín, Medellín, Colombia. Carrera 87 n. 30-65. Teléfono: 3405536. Correo electrónico: mluna@udem.edu.co. Orcid: http:// orcid.org/0000-0002-4679-2125

${ }_{* * * * * *}^{*}$ Ph.D. en conservación ambiental. Instructora. Centro de Diseño y Manufactura del Cuero. SENA (Servicio Nacional de Aprendizaje), Itagüí, Colombia. Calle 63 58B-03. Teléfono: 3008714507. Correo electrónico: idiazf@sena.edu.co 


\title{
Web portals for information management and enhancement of the co-creational experience
}

\begin{abstract}
Multiple strategies for a more open innovation have been put into place looking for a conjunct generation of value among different actors through the co-creation of experiences. Some are face-to-face techniques in which participants have to be focused in the same space-time scenario, while other use social media and web portals because of their potential for facilitating collaboration and co-creation naturally while also providing specialized tools according to the particular requirements of s project. This research shows a framework for the selection of a web portal for co-creation, facilitating thus the choice for the more suitable portal for a virtual open innovation process with clear criteria. On one hand, some preliminary results highlight that the adequate choice of the interaction tool in a process of open innovation is decisive for the success of the process. On the other internet is ratified (specifically, tools like business innovation portals) is an enhancer for the interchange of information because it facilitates the interaction among participants geographically scattered while at the same time offers the opportunity of asynchronous collaboration. With this feature time and space as face-to-face limitants are eliminated.
\end{abstract}

Keywords: information technologies and communication; open innovation; web portals.

\section{Portais web para gerir informação e potencializar a cocriação de experiência}

\begin{abstract}
Resumo
Múltiplas estratégias para potencializar a inovação aberta têm sido criadas buscando a geração conjunta de valor entre diversos atores por meio da cocriação de experiências. Algumas consistem em técnicas presenciais nas quais os interessados devem estar concentrados no mesmo lugar e tempo, enquanto outras usam as redes sociais e portais web porque facilitam a colaboração e a cocriação de uma maneira natural e, além disso, proporcionam ferramentas especializadas de acordo com os requisitos particulares de um projeto. Este trabalho mostra um referencial para a seleção de um portal web com fins de cocriação, o que facilita o trabalho de escolher, com critérios claros, a melhor ferramenta para um processo de inovação aberta virtual. Alguns resultados preliminares permitem tornar evidente que a seleção adequada da ferramenta de interação em um processo de inovação aberta é determinante para o sucesso do processo. Por outro lado, ratifica-se que a internet (pontualmente ferramentas como os portais empresarias de inovação) é uma potencializadora no intercâmbio de informação. Isso porque facilita a interação entre participantes distribuídos geograficamente e oferece possibilidades de colaborar de forma assincrônica. Com isso, são eliminadas as limitantes de tempo e espaço que são apresentados ao pensar em esquemas presenciais para cocriar.
\end{abstract}

Palavras-chave: tecnologias da informação e comunicação; inovação aberta; portais web; cocriação. 


\section{INTRODUCCIÓN}

La innovación hace parte de la tendencia global de las compañías en los sectores productivo, de servicios, de investigación y el sector gubernamental para acometer nuevos desarrollos que minimicen costos, reduzcan tiempo de fabricación, aumenten rentabilidad, y entreguen valor agregado a los usuarios de dichos productos o servicios [1].

Los modelos de innovación están basados en la generación de redes. Estos pueden ser claramente soportados a través del uso de internet, dado que es posible fortalecer la cocreación de valor en grupos masivos, donde incluso los actores pueden o no conocerse. Los servicios provistos por internet se perfilan como grandes potenciadores de los procesos de innovación ya que las contribuciones de los participantes pueden ser almacenadas, compartidas y discutidas de manera asíncrona, permitiendo el acceso en cualquier momento y en cualquier lugar. Particularmente los portales web especializados se perfilan como una opción bastante atractiva para facilitar la cocreación de valor, sin embargo, no se evidencia en la literatura alguna aproximación o iniciativa que facilite la selección sistemática de un portal para un ejercicio de cocreación específico, y se decide a partir de elementos subjetivos dada la falta de orientación.

El presente trabajo propone un marco de referencia de selección de portales web que apoyan la cocreación, para que, mediante unos requerimientos específicos asociados a un escenario puntual, un usuario pueda realizar una revisión y escoger, con criterios claros, cuál herramienta es óptima para atender sus necesidades. También se presenta un ejercicio preliminar para la validación del marco de referencia mediante la revisión de un conjunto de portales para cocrear.

El resto del artículo se estructura así: en la sección 1 se presenta el marco teórico, donde se definen conceptos básicos del trabajo desarrollado; en la sección 2 se enuncian algunos antecedentes y finaliza con la identificación de falencias; en la sección 3 se muestra el marco de referencia propuesto; en la sección 4 se presenta un ejercicio preliminar para la validación del marco de referencia propuesto; en la sección 5 se discuten los hallazgos; en la última sección se presentan las conclusiones.

\section{MARCO TEÓRICO}

\subsection{Innovación abierta}

De acuerdo al manual de Oslo, la innovación se define como la implementación de un producto (bien, servicio, proceso) nuevo o significativamente mejorado que se incorpora en el mercado [2] . Como parte de los modelos de últimas generaciones para innovar, 
se potencia el concepto innovación abierta, cuyo objetivo es combinar el conocimiento interno en las organizaciones con el conocimiento fuera de las mismas [3], por medio de las cooperación con clientes, aliados, e incluso competidores.

\subsection{Cocreación de valor}

La cocreación es definida como una forma de interacción y colaboración entre clientes, empleados, proveedores o cualquier participante en un proceso. Se trata de una estrategia para materializar la innovación abierta. Para lograrlo es posible aplicar técnicas con esquema presencial como Knowledge Safari, brainstorming, por mencionar algunas [4]. Un segundo tipo de cocreación es la mediada por las tecnologías de la información y la comunicación-TIC, en la cual el uso de las redes sociales y los portales web permite generar ideas sin requerir la concentración geográfica o temporal de los participantes [5].

\subsection{Portales web para potenciar la cocreación}

Este concepto hace referencia a espacios abiertos (o en ocasiones restringuidos) que sirven de plataforma para lanzar retos y necesidades que deben ser satisfechas por medio de un enfoque de innovación abierta. Gracias a ellos es posible que todo tipo de agentes (clientes, colaboradores internos y externos, e incluso competidores) se sumen a la construcción de la solución requerida [6]. Los portales web para la cocreación incorporan un conjunto de herramientas (tales como blogs, wikis, chats, foros, entre otros) que permiten a los usuarios interactuar con una comunidad y enriquecer las innovaciones.

\section{ESTADO DEL ARTE}

En esta sección se resumen algunos de los referentes encontrados en la literatura, cuyo objetivo es proponer buenas prácticas para el uso de herramientas TIC en el contexto de la cocreación [7]. En [8] se propone una red social para facilitar la colaboración creativa. Dentro de este estudio se involucraron cincuenta diseñadores de interfaces, arquitectos de información e ingenieros de software. A través de dicha red se hizo visible la información a todos los integrantes del equipo (antes, la baja interacción generaba este problema). La red social tiene un formato de blog, y permite compartir espacios de trabajo, además presenta una forma para votar los aportes de cada usuario (clasificándolos de acuerdo a su relevancia).

En [7] se estudian herramientas de interacción usadas cotidianamente en el departamento de policía de Honolulú (teléfono, correo electrónico y face to face). Posteriormente, se incluye una red social, con el objetivo de optimizar la comunicación 
entre funcionarios. La colaboración entre filiales y la interacción se incrementaron de manera notable. El blog se usa como herramienta básica de interacción. En [9] se propone GenSpace, una herramienta de colaboración en el ámbito de la divulgación científica, como potenciadora de la generación de nuevo conocimiento conjunto. En esta herramienta se usan: mensajería instantánea, redes de contactos a partir de sugerencias basadas en el perfil y clasificación de perfiles (básicos y expertos), de acuerdo al nivel de aportes e interacción.

En [10] se realiza un estudio profundo sobre las herramientas colaborativas disponibles en redes sociales, se encuentra que las más representativas son: correo electrónico, chat, foros, listas de discusión y distribución, microblogs, wikis. De otro lado, en [11] se diseña una herramienta colaborativa para apoyar la reducción de requisitos en diseño de software, mediante la cual se probó que la comunicación fue más efectiva y fluida y con mayor grado de trazabilidad, involucrando herramientas como blogs, espacios compartidos de trabajo, la posibilidad de asignación de etiquetas por cada proyecto, sistemas de premiación con puntajes y personalización de perfiles que incluyan información de las contribuciones de cada participante.

La tabla 1 muestra las herramientas propuestas para potenciar la cocreación al hacer uso de servicios en Internet.

Tabla 1. Herramientas de interacción mediadas por TIC identificadas en la literatura

\begin{tabular}{cl}
\hline Autor & \multicolumn{1}{c}{ Herramienta de interacción } \\
\hline$[8]$ & $\begin{array}{l}\text { Blogs, espacios compartidos de trabajo, asignación de etiquetas proyecto, mecanismos de premia- } \\
\text { ción con puntajes y personalización de perfiles que incluyan información de las contribuciones } \\
\text { de cada participante. }\end{array}$ \\
\hline$[11]$ & $\begin{array}{l}\text { Intercambio de mensajes de forma síncrona y asíncrona, sugerencias de contactos de acuerdo a } \\
\text { las preferencias del perfil personal de cada participante. }\end{array}$ \\
\hline$[10]$ & Correo electrónico, chat, foros, listas de discusión y distribución, microblogs, wikis. \\
\hline
\end{tabular}
Fuente: elaboración propia.

A partir de la revisión de la literatura se observan algunas oportunidades. En principio, las propuestas se enfocan al uso de servicios de internet como potenciadores de las interacciones entre equipos de personas que acometen retos. No obstante, en los procesos de innovación bajo enfoque de cocreación es influyente, además de conocer las técnicas y los métodos de interacción, el planteamiento de roles (cuáles actores deberían ser convocados), cuál fase o fases de la cadena de innovación se estaría impactando a través del uso de las TIC [2] y, finalmente, de qué manera se motivaría a los participantes a continuar [12]. A partir del estudio de portales web tratado en este 
artículo, será posible resolver tales interrogantes. De otro lado, no se evidencia alguna aproximación que permita, de acuerdo a unos requerimientos particulares, realizar la escogencia de un portal web que potencie la cocreación.

\section{SOLUCIÓN PROPUESTA}

En la figura 1 se presenta un marco de referencia para seleccionar un portal web como herramienta en un ejercicio de cocreación. Las subsecciones explican con más detalle cada paso dentro del proceso.

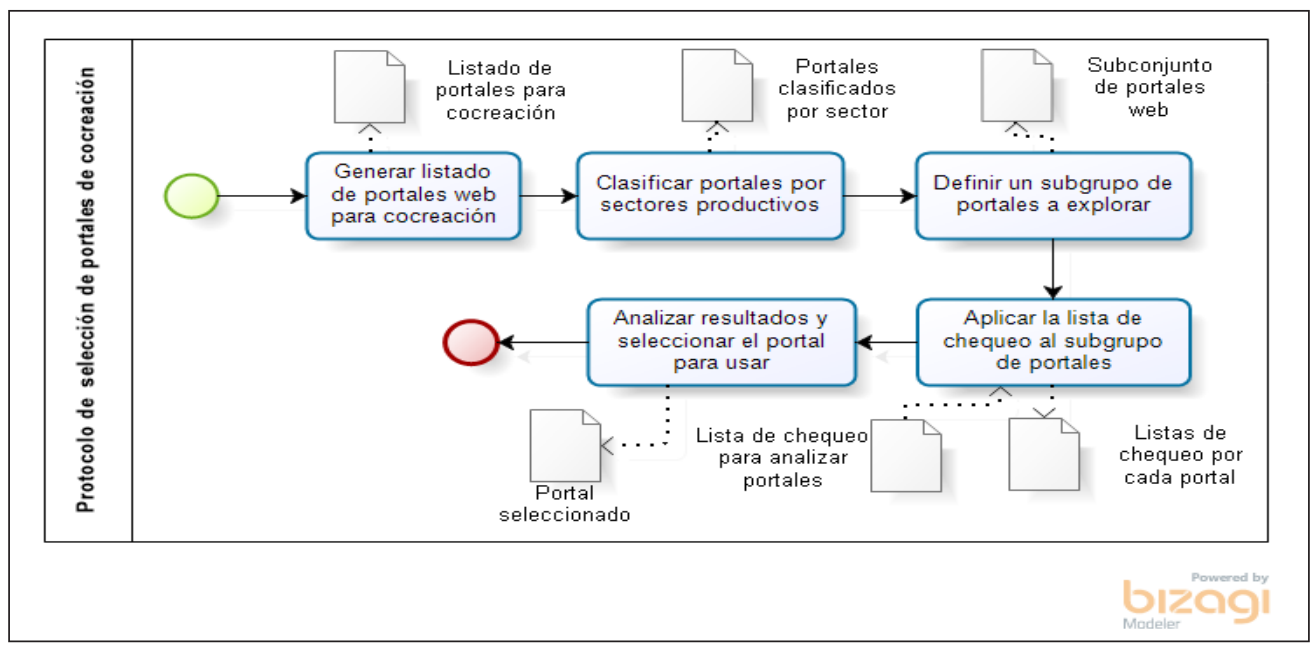

Figura 1. Marco de referencia propuesto para la escogencia de sitios web para cocreación Fuente: elaboración propia.

\subsection{Generación de un listado de portales web para cocreación}

En primera instancia, se propone recopilar una muestra significativa de sitios web para cocreación. Para el efecto se sugiere establecer un conjunto apropiado de descriptores de búsqueda tales como:

- (Co-creation OR open innovation) AND (platform OR web portal).

- Crowdsourcing AND ICT AND Tools.

- Idea AND Generation AND Platform.

- Collective Intelligence AND Website.

Finalizando esta fase se tendrá un inventario de los portales web para cocreación junto con el fin primario para el cual fueron concebidos. 


\subsection{Clasificación de portales por sectores productivos}

Se propone clasificar el inventario de la fase anterior acorde a los tipos de retos que se despliegan en cada sitio web (de acuerdo a un sector específico o sector productivo). En esta instancia, el usuario debe seleccionar cuál (o cuáles) de estos nichos de negocio son de su interés, y así filtrar los sitios apropiados para el ejercicio de cocreación que pretende realizar. Para facilitar el filtrado de portales de acuerdo a su nicho de negocio, se propone utilizar una herramienta de clasificación de texto. Se sugiere Uclassify, que entrega una ponderación de temáticas de negocio. Como producto de esta fase se tendrá un subconjunto de portales web a explorar y su clasificación por sector productivo.

\subsection{Aplicación de la lista de chequeo}

Como último paso del marco de referencia, se propone aplicar la lista de chequeo relacionada en la tabla 2. Este instrumento se fabricó con base en el estudio de referentes mostrado en la sección de estado del arte. En cada sitio seleccionado deben identificarse: herramientas, tipos de perfiles, formas de motivar a los participantes, entre otras. Con esta lista de chequeo se busca proporcionar, tal como se mencionó anteriormente, una forma argumentada de elegir el portal web a utilizar en un ejercicio de cocreación particular.

Tabla 2. Lista de chequeo para sitios web que apoyan la cocreación

\begin{tabular}{|c|c|c|}
\hline & Característica buscada & Descripción \\
\hline 1 & $\begin{array}{l}\text { Fases del proceso de } \\
\text { innovación que apoya } \\
\text { visiblemente el sitio o } \\
\text { plataforma }\end{array}$ & $\begin{array}{l}\text { Usando un conjunto de fases de un ciclo de innovación identificado se define } \\
\text { para cuáles es apropiado el sitio web bajo exploración. Se sugiere utilizar } \\
\text { la cadena de innovación propuesta por los autores de este artículo en una } \\
\text { publicación previa [13]. }\end{array}$ \\
\hline 2 & $\begin{array}{l}\text { Herramientas que usa } \\
\text { para generar interacción }\end{array}$ & $\begin{array}{l}\text { Se buscan estrategias tales como foros, blogs, videoconferencias, chats, entre } \\
\text { otros. }\end{array}$ \\
\hline 3 & $\begin{array}{l}\text { Mecanismos de } \\
\text { premiación }\end{array}$ & $\begin{array}{l}\text { Se identifican los esquemas usados para motivar a los participantes a contribuir } \\
\text { en un reto específico. }\end{array}$ \\
\hline
\end{tabular}

Fuente: elaboración propia.

Al aplicar el instrumento será posible tener claro, a partir de cada uno de los ítems evaluados, cuáles sitios son potencialmente útiles para acometer un proyecto de innovación abierta. Por ejemplo, si se desea cocrear en fases avanzadas de la cadena de innovación, sería posible identificar cuáles sitios tendrían soporte y cuáles no.

\section{EJERCICIO PRELIMINAR PARA LA VALIDACIÓN DEL FRAMEWORK PROPUESTO}

En esta sección se aplica el marco de referencia propuesto. Algunos indicadores de evaluación de los sitios son aportados en cuanto a perfiles, fases apoyadas, mecanismos de motivación, entre otros. 


\subsection{Generación de un listado de portales web para cocreación}

Al emplear los descriptores de búsqueda planteados anteriormente se logra la base de datos mostrada en la tabla 3 , la cual a su vez es insumo para la siguiente fase.

\section{Tabla 3. Sitios web que apoyan la cocreación mediada por TIC}

\begin{tabular}{|c|c|}
\hline Plataformas para potenciar $I+D$ & Servicios intermediarios para innovación abierta \\
\hline Innocentive - open innovation problem solving & $\begin{array}{l}\text { Big Idea Group - organize innovation contests and } \\
\text { idea hunts }\end{array}$ \\
\hline $\begin{array}{l}\text { IdeaConnection - idea marketplace and problem } \\
\text { solving }\end{array}$ & Skild - organize innovation quests \\
\hline Yet2.com - IP market place & $\begin{array}{l}\text { Pharmalicensing - open innovation for the life } \\
\text { sciences }\end{array}$ \\
\hline PRESANS (beta) - connect and solve R\&D problems & Chaordix - crowdsourcing engine for innovation \\
\hline Hypios - online problem solving & DataStation - complete innovation platform \\
\hline Innoget - research intermediary platform & Cocreación creativa \\
\hline One Billion Minds - online (social) challenges & Spreadshirt - shirt community \\
\hline NineSigma - technology problem solving & Threadless - create and sell your t-shirts \\
\hline Ideaken - collaborative crowdsourcing & cafepress - shop, create or sell what's on your mind \\
\hline $\begin{array}{l}\text { Innovation-community.de - Community of innova- } \\
\text { tors \& creators. }\end{array}$ & zazzle - create and sell products \\
\hline $\begin{array}{l}\text { Plataformas para mercadeo, diseño y generación de } \\
\text { ideas }\end{array}$ & CreateMyTattoo - crowdsourced tattoo design \\
\hline CMNTY Corporation - community co-creation & Sellaband - crowdfunded bands \\
\hline Innovation Exchange - open innovation market place & Artistshare - fans funding new artists \\
\hline Idea Bounty - crowdsourcing ideas & Quirky - community product development \\
\hline Guerra Creativa - crowdsourcing anything creative & jovoto - co-creation \& mass collaboration \\
\hline Brand Tags - tagging brands & Dream Heels - design your dream heels \\
\hline Battle of concepts - student challenges & Mookum- co-creating lifestyle \& interior products \\
\hline crowdSPRING - creative designs & $\begin{array}{l}\text { Quirky + GE - co-creating platform by quirky \& } \\
\text { General Electric }\end{array}$ \\
\hline $\begin{array}{l}\text { BootB.com - custom creative ideas for any creative } \\
\text { need }\end{array}$ & Userfarm - co-creating platform for Video makers \\
\hline
\end{tabular}

12designer - marketplace for creative solutions

Plataformas de cocreación de productos impulsadas

por corporaciones

LeadVine - crowdsourcing lead generation

Fiat Mio - create a car 


\begin{tabular}{|c|c|}
\hline Plataformas para potenciar $I+D$ & Servicios intermediarios para innovación abierta \\
\hline 99designs - pioneer in design crowdsourcing & BMW Customer Innovation Lab - in german \\
\hline Edge Amsterdam - elite sourcing platform & Kraft - innovate with Kraft \\
\hline OpenIDEO - collaborative design platform & InnovationJam* - IBM's idea generation project \\
\hline $\begin{array}{l}\text { Challenge.gov - crowdsourcing for government } \\
\text { problems }\end{array}$ & Dell IdeaStorm - external idea sourcing \\
\hline eYeka - the co-creation community & Betavine - Vodafone’s mobile app community \\
\hline Spigit - social innovation platform & $\begin{array}{l}\text { My Startbucks Idea - shaping the future of } \\
\text { Starbucks }\end{array}$ \\
\hline Cognistreamer - open innovation platform & Ideasbrewery - Heineken Ideas Brewery \\
\hline Zooppa - Branding/marketing platform & Connect + Develop - Innovation platform by P\&G \\
\hline Plataformas para inteligencia colectiva & Plataformas para diseño de marca \\
\hline Lumenogic - collective intelligence markets & Peugeot - Peugeot’s design contest \\
\hline Ushahidi - crowdsourcing crisis information & Fluevog - open shoe design \\
\hline Kaggle - data mining and forecasting & BurdaStyle - open source sewing \\
\hline We Are Hunted - the online music chart & $\mathrm{P} 2 \mathrm{P}$ \\
\hline $\begin{array}{l}\text { Google Image Labeler - crowdsourced image } \\
\text { labeling }\end{array}$ & Funding Circle - p2p lending \\
\hline Plataformas de cocreación para freelancers & Linux - open source software \\
\hline $\begin{array}{l}\text { TopCoder - competition-based software } \\
\text { crowdsourcing }\end{array}$ & Wikipedia - peer produced encyclopedia \\
\hline Spudaroo - crowdsourcing copywriting & Yahoo Answers - crowdsourced Q\&A \\
\hline Clickworker - small online task solving & A Swarm of Angels - creating a $£ 1$ million film \\
\hline Amazon Mechanical Turk - low-cost crowdsourcing & Cocreación abierta al público en general \\
\hline Software para innovación abierta & Science Commons- generic license agreements \\
\hline Imaginatik - collective intelligence software & Picnic Green Challenge - ideas to save the planet \\
\hline $\begin{array}{l}\text { Napkin Labs - connect with consumers, experts, } \\
\text { employees }\end{array}$ & Fold it - solve puzzles for science \\
\hline Venture Spirit - gamification platform & Galaxy Zoo - discovering the universe \\
\hline
\end{tabular}

\subsection{Clasificación de portales por sectores productivos}

Tomando el inventario de la tabla anterior, y para efectos del caso de estudio, se desea filtrar los portales web para cocrear en el sector de las TIC. No interesan, por ejemplo, aquellos sitios destinados a proponer innovaciones de corte social. Como se 
sugirió en el marco de referencia, se usa una herramienta de clasificación de texto para evaluar cada uno de los portales recopilados en la tabla 3. En la figura 2 se muestra un ejemplo de clasificación para un sitio de cocreación particular. En la tabla 4 se muestran los portales seleccionados para ser estudiados, de acuerdo a su foco temático orientado a TIC (sumando los porcentajes de tecnologías de información con los de telecomunicaciones).

Tabla 4. Sitios seleccionados a estudiar, de acuerdo a su contenido (orientado a TIC)

\begin{tabular}{|c|c|c|c|}
\hline Research \& Development platforms & $\%$ TIC & Research \& Development platforms & $\%$ TIC \\
\hline $\begin{array}{l}\text { Google Image Labeler - crowdsourced } \\
\text { image labeling }\end{array}$ & $85 \%$ & $\begin{array}{l}\text { TopCoder - competition-based } \\
\text { software crowdsourcing }\end{array}$ & $41 \%$ \\
\hline $\begin{array}{l}\text { InnovationJam* - IBM’s idea generation } \\
\text { project }\end{array}$ & $85 \%$ & Idea Bounty - crowdsourcing ideas & $38 \%$ \\
\hline Fold it - solve puzzles for science & $84 \%$ & $\begin{array}{l}\text { Zooppa - Branding/marketing } \\
\text { platform }\end{array}$ & $36 \%$ \\
\hline $\begin{array}{l}\text { Betavine - Vodafone’s mobile app } \\
\text { community }\end{array}$ & $70 \%$ & $\begin{array}{l}\text { DataStation - complete innovation } \\
\text { platform }\end{array}$ & $35 \%$ \\
\hline $\begin{array}{l}\text { Challenge.gov - crowdsourcing for } \\
\text { government problems }\end{array}$ & $62 \%$ & $\begin{array}{l}\text { NineSigma - technology problem } \\
\text { solving }\end{array}$ & $33 \%$ \\
\hline $\begin{array}{l}\text { Imaginatik - collective intelligence } \\
\text { software }\end{array}$ & $62 \%$ & $\begin{array}{l}\text { Quirky + GE - co-creating platform } \\
\text { by quirky \& General Electric }\end{array}$ & $30 \%$ \\
\hline $\begin{array}{l}\text { Science Commons-generic license } \\
\text { agreements }\end{array}$ & $61 \%$ & $\begin{array}{l}\text { Dell IdeaStorm - external idea } \\
\text { sourcing }\end{array}$ & $29 \%$ \\
\hline Spigit - social innovation platform & $60 \%$ & $\begin{array}{l}\text { CMNTY Corporation - community } \\
\text { co-creation }\end{array}$ & $25 \%$ \\
\hline Innoget - research intermediary platform & $57 \%$ & Venture Spirit - gamification platform & $25 \%$ \\
\hline $\begin{array}{l}\text { Cognistreamer - open innovation } \\
\text { platform }\end{array}$ & $46 \%$ & $\begin{array}{l}\text { IdeaConnection - idea marketplace } \\
\text { and problem solving }\end{array}$ & $24 \%$ \\
\hline
\end{tabular}

Fuente: elaboración propia.

\subsection{Aplicación de la lista de chequeo}

Una vez filtrados los portales a explorar con mayor detalle, se aplicó la lista de chequeo mostrada en la tabla 2. Los sitios preseleccionados (tabla 4) fueron revisados para buscar los parámetros de la lista de chequeo, y así especificar sus características. En términos generales, puede mencionarse que todas las plataformas exploradas basan su filosofía de operación en retos. Los retos son necesidades puntuales que tienen las compañías, las cuales deben ser satisfechas a través de una innovación, en este caso, cocreada. Una vez el reto se publica en el sitio web, las condiciones del mismo, el tipo de premiación, fechas de entrega y condiciones generales son fijadas para que los 
agentes interesados aporten sus iniciativas en orden de resolver la problemática. En los siguientes apartados se mostrarán algunos hallazgos particulares sobre los portales web explorados.

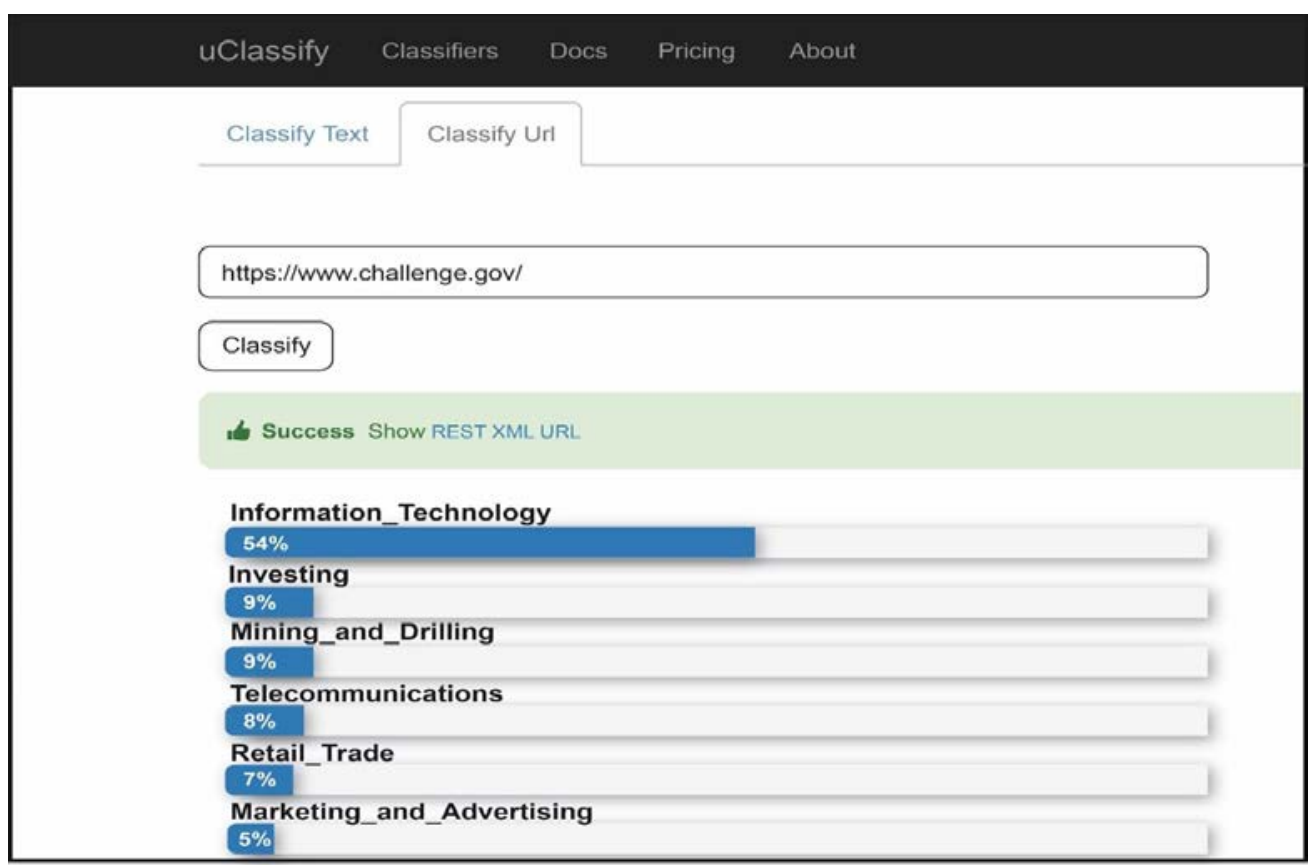

Figura 2. Ejemplo de clasificación de sitios web de cocreación de acuerdo a sector productivo Fuente: [36].

\subsubsection{Fases de innovación apoyadas}

La tabla 5 muestra el porcentaje de sitios que apoyan visiblemente cada una de las fases de la cadena de innovación en el modelo tomado como guía [13]. Los portales web explorados apoyan en su mayoría fases tempranas de la cadena de innovación (la totalidad de las plataformas soporta las fases de identificación de oportunidades y generación de ideas), pero existe una muy baja participación en fases avanzadas tales como las pruebas, la distribución y la operación. No se encuentran portales web para soportar cocreación en fases como la medición de impacto en el mercado.

Tabla 5. Fases de innovación apoyadas por los sitios web estudiados

\begin{tabular}{lcllc}
\hline \multicolumn{1}{c}{ Fases } & Porcentaje & Fases & Porcentaje \\
\hline Identificación de oportunidades & $100 \%$ & Diseño & $30 \%$ \\
\hline Generación de ideas & $100 \%$ & Construcción & $30 \%$ \\
\hline
\end{tabular}


Liliana González P., Mauricio González P., Manuel E. Anaya T., Mario A. Luna-del Risco e Isabel Díaz-Forero

\begin{tabular}{lclc}
\hline \multicolumn{1}{c}{ Fases } & Porcentaje & Fases & Porcentaje \\
\hline Propuesta de producto/servicio & $70 \%$ & Pruebas & $30 \%$ \\
\hline Investigación & $40 \%$ & Operación & $10 \%$ \\
\hline Definición de requerimientos & $30 \%$ & Distribución & $10 \%$ \\
\hline Modelado conceptual & $30 \%$ & Evaluación de impacto & $0 \%$ \\
\hline
\end{tabular}

Fuente: elaboración propia.

\subsubsection{Herramientas de interacción encontradas}

La figura 3 muestra las herramientas para generar interacción dentro de los portales web explorados. Los hallazgos coinciden con la literatura al respecto (tabla 1).

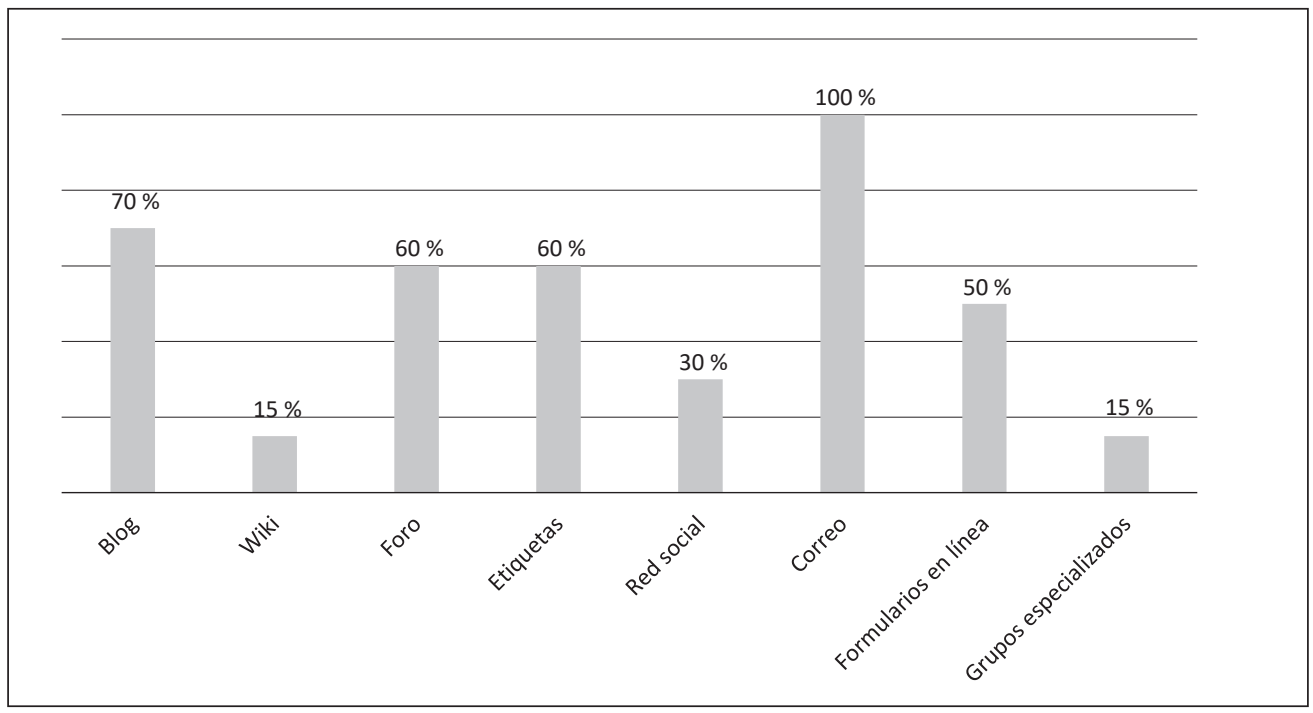

Figura 3. Herramientas de interacción usadas en los sitios web de cocreación explorados Fuente: elaboración propia.

Se puede observar que, contrario a lo que se intuiría, las redes sociales no juegan un rol protagónico en las interacciones entre los interesados en el proceso, debido a que en la mayoría de los casos no es posible tener vínculos directos con otros participantes, solamente entre un participante particular y la compañía que desea innovar usando cocreación.

\subsubsection{Mecanismos de motivación}

Como se muestra en la tabla 6, el mecanismo más popular para motivar a los participantes en los portales web explorados, es ofrecer una recompensa económica, en caso 
de que la idea de un nuevo producto o servicio sea tomada por la compañía que impulsa la innovación. Sin embargo, otros portales motivan a los participantes externos por medio del ofrecimiento de patentes conjuntas entre el creador de la idea y la compañía.

Tabla 6. Mecanismos de motivación para los participantes

\begin{tabular}{lclc}
\hline \multicolumn{1}{c}{ Mecanismos de premiación } & Porcentaje & Mecanismos de premiación & Porcentaje \\
\hline Patentes & $10 \%$ & Mejorar capacidades/autoaprender & $10 \%$ \\
\hline Premiación monetaria & $80 \%$ & Reputación/puntuación & $40 \%$ \\
\hline \multicolumn{4}{c}{ Fuente: elaboración propia. }
\end{tabular}

\subsubsection{Mecanismos para realizar seguimiento a las ideas generadas}

En la tabla 7 se muestra el flujo que siguen las ideas generadas por los participantes en la innovación cocreada pasando por diferentes estados que muestran su grado de madurez y ejecución.

Tabla 7. Información del estado de las ideas generadas en los sitios web que apoyan la cocreación

\begin{tabular}{lclc}
\hline \multicolumn{1}{c}{ Estado de los aportes } & Porcentaje & \multicolumn{1}{c}{ Estado de los aportes } & Porcentaje \\
\hline Recolección de ideas & $100 \%$ & Puesto en acción & $20 \%$ \\
\hline Trabajo en progreso & $20 \%$ & Ejecutado & $40 \%$ \\
\hline
\end{tabular}

Fuente: elaboración propia.

Cuando una necesidad es publicada por una empresa que necesita una idea innovadora para resolverla, un reto es abierto, y cualquier interesado puede aportar ideas para la solución de la problemática. En este momento particular se informa a los participantes que el reto está en la fase de recolección de ideas. Si una idea particular es tomada como la mejor solución a la necesidad expuesta, la empresa informa esta situación mediante el estado trabajo en progreso. Una vez el producto o servicio fruto de la idea generada en principio es liberado al mercado, un estatus adicional se indica como puesto en acción. Finalmente, un estatus de ejecutado indica al dueño de la idea inicial que la innovación fue exitosa. En este caso solamente un $20 \%$ de las plataformas consultadas indican el avance de las ideas generadas inicialmente, manifestando una falta de realimentación de la empresa que ejecuta la innovación hacia los creadores iniciales de la idea, lo cual podría redundar en desmotivación para participar en futuros procesos, o en la no fidelización en la cocreación.

\subsubsection{Tipos de coordinación}

La forma en la cual las ideas son seleccionadas para resolver una determinada problemática varía de acuerdo al sitio web. En tal caso, se han detectado dos tipos 
de coordinación en la cual la decisión de clasificar una idea reposa en la compañía (centralizada) o distribuida (existen agentes externos que tienen injerencia en la decisión). El 70 \% de los sitios estudiados implementa coordinación centralizada, el 20 \% coordinación distribuida y el 10 \% coordinación mixta. Si bien la coordinación de tipo centralizado es más común que la de tipo distribuido, algunos sitios permiten ambos tipos de coordinación. En la coordinación distribuida, particularmente, los filtros de ideas a poner en marcha se realizan de tal forma que las decisiones también de ejecutar o no una iniciativa también son cocreadas.

\subsubsection{Perfiles/roles}

En la tabla 8 se muestran los tipos de perfiles identificados en los portales web estudiados. Dichos perfiles serán de utilidad cuando se requiera recapitular los tipos de participantes más comunes en un proceso de innovación bajo enfoque de cocreación con soporte de las TIC.

Tabla 8. Tipos de perfiles/roles identificados en las plataformas estudiadas

\begin{tabular}{lclc}
\hline \multicolumn{1}{c}{ Perfiles } & Porcentaje & \multicolumn{1}{c}{ Perfiles } & Porcentaje \\
\hline Creativo estándar & $100 \%$ & Administrador del sitio & $100 \%$ \\
\hline Creativo experto & $10 \%$ & Jurado externo & $10 \%$ \\
\hline Director del reto & $10 \%$ & Empresa que publica el reto & $40 \%$ \\
\hline Asesor del reto & $10 \%$ & Competidor & $10 \%$ \\
\hline Moderador & $20 \%$ & Proveedor & $10 \%$ \\
\hline
\end{tabular}

Fuente: elaboración propia.

Los porcentajes mostrados hacen referencia a la presencia de un tipo de perfil particular en los sitios web explorados. Los participantes más frecuentes son los creativos estándar. Esto podría interpretarse desde el punto de vista de que las compañías que desean innovar no tienen comunicación visible por medio de las herramientas web analizadas con otras compañías, tales como proveedores, socios estratégicos o, incluso, la competencia. Es natural el resultado porque en principio la cocreación solo se da con clientes u otros particulares que deseen obtener algún beneficio. A continuación, se explica a grandes rasgos el papel que juega cada uno de los tipos de los perfiles identificados en los portales explorados:

- Creativo estándar: participante que está atento a las necesidades de la empresa con la cual está cocreando en orden de realizar aportes. 
- Creativo experto: debido a que sus aportes han sido exitosos, este tipo de participante tiene una reputación más alta y tiene permisos adicionales como acceder a proyectos de mayor retribución.

- Directores, asesores y moderadores del reto: cuando el esquema del sitio web está orientado tener una coordinación distribuida (la clasificación y aceptación de ideas será realizada por actores por fuera de las empresas), cualesquiera de estos tres perfiles pueden aparecer. Los primeros se encargan de administrar los recursos disponibles, los segundos entregarán conocimiento técnico en el área de conocimiento particular y los terceros velan que por la normatividad y reglas del reto sean cumplidas.

- Jurado externo: en algunos casos puede requerirse de la ayuda de un agente externo a la organización para arbitrar la escogencia, pertinencia y madurez de las ideas.

- Administrador del sitio: en algunos casos se encontró que la empresa que posee la infraestructura del sitio web arrienda a terceros sus recursos para que estos puedan cocrear. De tal manera se identificó este perfil, el cual se encarga de establecer permisos para acceder a los repositorios de servicios a las compañías que subarriendan la plataforma.

- Empresa que publica el reto: en el caso de que la plataforma pueda ser arrendada, este perfil será personificado por el tercero que desea acceder a la plataforma.

- Competidor: aquella compañía que desee asumir como participantes a sus competidores (para desarrollar estándares conjuntos en un área del conocimiento particular, unificar tarifas de servicios o productos, entre otros). Este perfil es quizás el menos popular dentro de los sitios estudiados, posiblemente porque la inclusión de la competencia en innovaciones cocreadas puede ser sensible a problemas de propiedad intelectual.

- Proveedor: se identificó un caso en el cual, en la situación hipotética de requerir apoyo desde la fase de concepción del producto, la empresa que requiere la solución puede invitar a sus proveedores para que interactúen con los demás participantes.

\section{DISCUSIÓN DERESULTADOS}

A partir del ejercicio preliminar de validación presentado anteriormente se pudo encontrar que:

- $\quad$ Hay poca evidencia de que los portales explorados apoyen fases avanzadas de la cadena de innovación. 
- La motivación que tienen los agentes externos a las compañías al cocrear tiene su núcleo principal en las recompensas económicas cuando la idea genera un producto o servicio y deriva en ganancias. Sin embargo, en portales de cocreación al servicio de comunidades de software libre, la motivación va orientada a la reputación que se gana al realizar aportes significativos para la solución de un problema.

- Los participantes dentro de los sitios web estudiados son, en gran medida, los clientes de la compañía que publica el reto. Sin embargo, al cocrear existen otros tipos de participantes, que pueden ser personas o instituciones, los cuales pueden apoyar tanto fases tempranas como avanzadas de la cadena de innovación.

- La mayor parte de los sitios que apoyan la cocreación no informan a los participantes acerca del avance de las ideas generadas. Esto redunda en que la cocreación está presentándose de manera parcial, coartándose el diálogo bilateralmente.

\section{CONCLUSIONES}

En el presente trabajo se propuso un marco de referencia para el estudio y la selección de portales web de cocreación a partir de un conjunto de parámetros claros. También se evidenció, a partir de la literatura, la potencia que el internet tiene sobre herramientas empresariales útiles para llevar a cabo procesos de innovación.

El marco de referencia se configura a partir de tres fases: generación de un listado de portales, clasificación por sectores productivos y aplicación de un lista de chequeo provista para analizar los portales. Empleando esta propuesta es posible

- Reconstruir un conjunto de buenas prácticas que ayuden a construir colaborativamente ideas y soluciones a retos específicos.

- Identificar los roles que participan y se aúnan para permitir la generación exitosa de ideas. Es evidente que en la cocreación no solo participan aquellos que generan ideas, sino otros mediadores que facilitan las interacciones.

- Entender el ciclo de innovación y las fases donde es útil incorporar el concepto de cocreación, y más allá de esto, identificar los portales web que facilitan la interacción.

- Conocer mecanismos para motivar a los interesados en cocrear valor.

- Analizar la forma de hacer seguimiento a las ideas generadas para mejorar las interacciones y la motivación.

El marco de referencia puede ser usado de manera sencilla y fiable, de acuerdo a unos parámetros de entrada en cada fase y los hallazgos permitirán elegir la mejor opción de acuerdo a las necesidades puntuales. 
En este artículo se demostró que el internet a través de herramientas como los portales web es un potenciador de procesos de innovación, ya que facilita la interacción de participantes distribuidos geográficamente, y ofrece posibilidades de colaborar de forma asíncrona. Con esto se eliminan las limitantes de tiempo y espacio que se presentan al pensar en esquemas presenciales para cocrear.

Como trabajo futuro, se puede generar una herramienta que permita tomar las mejores prácticas de cada una, así como incorporar las herramientas que aún no se usan masivamente en este dominio (tales como las redes sociales). También se desea emplear este protocolo en ejercicios empresariales reales para validar en un caso real su efectividad.

\section{REFERENCIAS}

[1] J. Eckhardt et al., "Open innovation, information, and entrepreneurship within platform ecosystems,” Strategic Entrepreneurship Journal, vol. 12, pp. 369-391, 2018.

[2] OECD y Eurostat, Oslo Manual 2018: Guidelines for Collecting, Reporting and Using Data on Innovation, 4. ${ }^{\text {a }}$ ed., Luxemburgo: OECD Publishing, 2018, 258 p.

[3] H. Chesbrough, Open innovation: The new imperative for creating and profiting from technology, Boston:Harvard Business School Press, 2006, 278 p.

[4] A. Samad et al., Hands-On Knowledge Co-Creation and Sharing: Practical Methods and Tecniques. Stuttgart: KnowledgeBoard, 2007, 584 p.

[5] L. González et al., "Method of interaction in open innovation processes incorporating ubiquitous environments and web 2.0 social networks: A baseline architecture,” presentado en 8th Iberian Conference on Information Systems and Technologies (CISTI), Lisboa, 2013.

[6] J. Rowley et al., "Customer community and co-creation: a case study,” Marketing Intelligence and Planning, vol. 25, pp. 136-146, 2007.

[7] M. Colombo et al., "Open innovation and within-industry diversification in small and medium enterprises: The case of open source software firms," Research Policy, vol. 43, n. ${ }^{\circ}$ 5, pp. 891-902, 2016.

[8] J. Perry-Smith y P. Mannucci, "From creativity to innovation: The social network drivers of the four phases of the idea journey,” Academy of Management Review, vol. 42, n. ${ }^{\circ}$, pp. 53-79, 2017.

[9] N. Oostervink et al., "Knowledge sharing on enterprise social media: Practices to cope with institutional complexity," Journal of Computer-Mediated Communication, vol. 21, n. ${ }^{\circ}$, pp. 156-176, 2016.

[10] M. Allen, “Web 2.0: An argument against convergence,” Media Convergence and Deconvergence, vol. 30, n. ${ }^{\circ}$, pp. 177-196, 2017. 
[11] S. Lim, A. Finkelstein, "StakeRare: using social networks and collaborative filtering for large-scale requirements elicitation,” IEEE transactions on software engineering, vol. 38, n. ${ }^{\circ}$, pp. 707-735, 2012.

[12] Päivi, H. and Antikainen, M. J., “Co-creating a digital service for small business owners’ finance management,” Journal of Innovation Management, 3, n. 3, pp. 57-70, 2015.

[13] L. González et al., "Proposal of a model of innovation process with focus in co-creation,” en Innovative ways of knowledge representation and management, C. Zapata y G. González, eds., pp. 165-178, Medellín: Sello Editorial Universidad de Medellín, 2012. 